\title{
Quantification of diffusion-weighted images (DWI) and apparent diffusion coefficient maps (ADC) in the detection of acute stroke
}

\author{
P. Karina Tulipano ${ }^{\mathrm{a}}$, William S. Millar ${ }^{\mathrm{b}}$, Celina Imielinska ${ }^{\mathrm{c}}$, Xin Liu ${ }^{\mathrm{a}}$, Joel Rosiene ${ }^{\mathrm{e}}$, Anthony L. \\ D'Ambrosio $^{\mathrm{d}}$ \\ ${ }^{a}$ Department of Biomedical Informatics, Columbia University, New York, NY 10032 \\ ${ }^{\mathrm{b}}$ Department of Radiology, Columbia University, New York, NY 10032 \\ ${ }^{\mathrm{c}}$ Department of Computer Science, Columbia University \\ ${ }^{\mathrm{d}}$ Department of Neurological Surgery, Columbia University, New York, NY 10032 \\ ${ }^{e}$ Department of Mathematics and Computer Science, Eastern Connecticut State University, CT
}

\begin{abstract}
Magnetic resonance (MR) imaging is an imaging modality that is used in the management and diagnosis of acute stroke. Common MR imaging techniques such as diffusion weighted imaging (DWI) and apparent diffusion coefficient maps (ADC) are used routinely in the diagnosis of acute infarcts. However, advances in radiology information systems and imaging protocols have led to an overload of image information that can be difficult to manage and time consuming. Automated techniques to assist in the identification of acute ischemic stroke can prove beneficial to 1) the physician by providing a mechanism for early detection and 2) the patient by providing effective stroke therapy at an early stage. We have processed DW images and ADC maps using a novel automated Relative Difference Map (RDM) method that was tailored to the identification and delineation of the stroke region. Results indicate that the technique can delineate regions of acute infarctions on DW images and ADC maps. A formal evaluation of the RDM algorithm was performed by comparing accuracy measurements between 1) expert generated ground truths with the RDM delineated DWI infarcts and 2) RDM delineated DWI infarcts with RDM delineated ADC infarcts. The accuracy measurements indicate that the RDM delineated DWI infarcts are comparable to the expert generated ground truths. The true positive volume fraction value (TPVF), between RDM delineated DWI and ADC infarcts, is nonzero for all cases with an acute infarct while the value for non-acute cases remains zero.
\end{abstract}

Keywords: computer-aided diagnosis, magnetic resonance, stroke, neurological, image segmentation, evaluation of segmentation.

\section{INTRODUCTION}

Magnetic resonance (MR) imaging has been used increasingly in the management of stroke. In particular, diffusion weighted imaging (DWI) is used to detect stroke in the early acute ischemic phase, when treatment of stroke can be effective. It has been shown that DWI has a high sensitivity and specificity for acute infarction and can differentiate acute stroke from other pathological conditions [1,2]. Although the absolute intensities of the DW images are useful, the diffusion constant at each pixel in the image may be affected by other factors such as heavy $\mathrm{T} 2$ weighting and

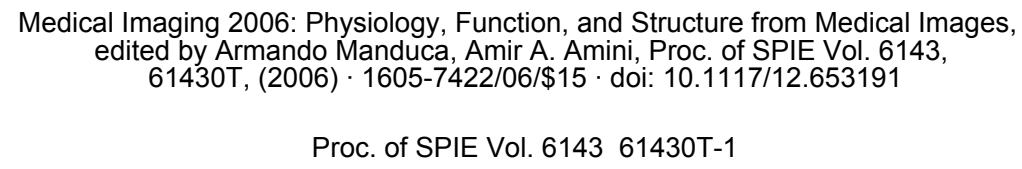


gradient strength. A better measure of diffusion is actually determined by the degree of signal decay (taken as the slope of the decay) [3]. This measure is then displayed in the form of an apparent diffusion coefficient (ADC) map. Radiologists use DWI and ADC maps in their final clinical diagnosis since DWI together with ADC maps can best verify the presence or absence of acute infarcts. While DWI has increased intensity in the infarction area, ADC maps depict the area as hypointense. On DWI, some regions with normal diffusion coefficient can also appear as hyperintense. The ADC maps can confirm the presence or absence of an acute infarct by depicting those areas of true diffusion while eliminating T2 and other factors, which may affect the signal. A true acute infarct with high signal intensity on DWI will remain hypointense on ADC map [4]. However, ADC maps are usually not used alone because of poor signal contrast. Hypointense infarcts on ADC maps tend to be less apparent to the radiologist [4]. Thus, DWI and ADC maps are used as complementary techniques in clinical practice.

Other imaging techniques have been also used in the detection of acute stroke. Conventional MR and Computed Tomography (CT) imaging lack the sensitivity needed to detect the early stages of stroke. A previous study indicates that these techniques show up to a $50 \%$ sensitivity in the hyperacute stroke period (typically within $0-6$ hours after the onset of symptoms) [5]. Another imaging technique, magnetic resonance perfusion (MRP), can also be used in the early stage of stroke detection. MRP can detect alterations in blood flow, indicating areas of early reversible and irreversible ischemic injury [6]. However, MRP has limitations when compared to DWI. MRP is an invasive technique requiring patients to be injected with a susceptibility contrast agent while DWI takes advantage of the intrinsic signal from the diffusional properties of molecules within the tissue. DWI is a safer technique that can be used to image all stroke patients without further patient risk. In addition, DWI is an overall faster procedure than MRP. While both MRP and DWI can take 60 seconds for scanning, MRP involves patient preparation (intravenous insertion and bolus injection times) and additional post processing time.

Despite this limitation, MRP can be used in conjunction with DWI in early patient stroke treatment and management. It has been shown that initial areas of small DWI abnormality can enlarge to larger volumes of reduced perfusion as indicated by MRP [7]. Thus, the use of both DWI and MRP may assist in the predication of patients with infarct enlargement over time. DWI and MRP can differentiate between tissue at risk for additional ischemic damage and tissue, which has already undergone maximal ischemic damage [8]. Identification of such regions can provide a mechanism for early, targeted treatment while also reducing patient exposure to unnecessary treatment involving invasive thrombolytic drugs and procedures.

The final clinical diagnosis of acute stroke involves the analysis of several different sets of images. The radiologist is confronted with the challenge of organizing and analyzing large volume of images in the decision making process. Automated techniques that can assist in the detection of acute infarcts from both DWI and ADC maps can potentially reduce the time between image acquisition and final diagnosis. Other research studies have been performed with DWI and ADC maps using an automated, multidimensional feature-based method that incorporates a unified segmentation procedure [8]. Adaptive segmentation techniques have also been previously used to determine brain and infarct volumes [9].

In this paper, we propose a novel automated segmentation method based on the relative difference map (RDM) method [10,11], which has been tailored to quantify and delineate acute infarct regions from DWI and ADC maps. We perform a formal evaluation of the RDM segmentation algorithm to assess the accuracy of the method. Expert generated ground truth is compared to RDM 
delineated DWI infarcts. In addition, accuracy parameters, [14], (true positive volume fraction (TPVF), false positive volume fraction (FPVF), false negative volume fraction (FNVF)) were computed for RDM delineated DWI infarcts as compared with RDM delineated ADC infarcts. While we demonstrate the potential of the technique, fully developed computer assisted methods to aid diagnosis of stroke will have to be thoroughly validated in clinical studies.

\section{METHODS}

\subsection{Image Acquisition and Selection}

DWI images and ADC maps were selected from several case studies. The images were acquired using a 1.5-T whole-body system (SIGNA EXCITE, GE Medical Systems, Milwaukee, WI). DW imaging was performed by using a single-shot spin-echo echo-planar imaging sequence. The imaging parameters for $b=1000 \mathrm{~s} / \mathrm{mm}^{2}$ images were as follows: TR/TE/NEX of 8000/105/1, a FOV of $22 \times 22 \mathrm{~cm}$, an image matrix of $256 \times 256$ pixels, a slice thickness of $5 \mathrm{~mm}$ with a 0 -mm gap. ADC maps were automatically generated using GE Signa Systems FuncTool software.

Seven cases were selected for this study (210 images). A total of seven image sets (DWI \& ADC) are presented in this paper. Image sets 1-6 are a representative collection of the most commonly encountered infarct patterns. Image set 7 represents a condition that mimics the presence of an acute infarct. Figure 1 (a-n) depicts both DWI and ADC maps for each of the infarct patterns described in Table 1.

\begin{tabular}{|l|l|l|l|}
\hline Image Set \# & Pattern \# & Description & Figure \# \\
\hline 1 & 1 & $\begin{array}{l}\text { Cortical infarct in the posterior cerebral artery (PCA) territory } \\
\text { involving the medial temporal and medial occipital lobes }\end{array}$ & Figure 1 (a) \& (b) \\
\hline 2 & 2 & Centrum semi ovale right infarct & Figure $1(\mathrm{c}) \&(\mathrm{~d})$ \\
\hline 3 & 3 & $\begin{array}{l}\text { Large territorial infarct in the left middle cerebral artery (MCA) } \\
\text { territory }\end{array}$ & Figure 1 (e) \& (f) \\
\hline 4 & 4 & Internal border zone, deep penetrating infarct & Figure $1(\mathrm{~g}) \&(\mathrm{~h})$ \\
\hline 5 & 5 & $\begin{array}{l}\text { Large territorial infarct spanning two territories, middle cerebral } \\
\text { artery (MCA) with some anterior cerebral artery (ACA) involvement, } \\
\text { possibly embolic }\end{array}$ & Figure 1 (i) \& (j) \\
\hline 6 & 6 & Small pontine infarct & Figure $1(\mathrm{k}) \&(\mathrm{l})$ \\
\hline 7 & N/A & Transient Ischemic Attack (TIA) & Figure $1(\mathrm{~m}) \&(\mathrm{n})$ \\
\hline
\end{tabular}

Table 1. Descriptions of the various image sets selected for processing with the RDM algorithm.

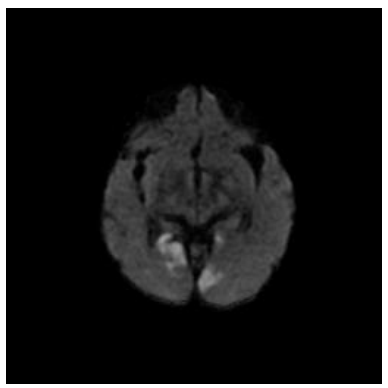

(a)

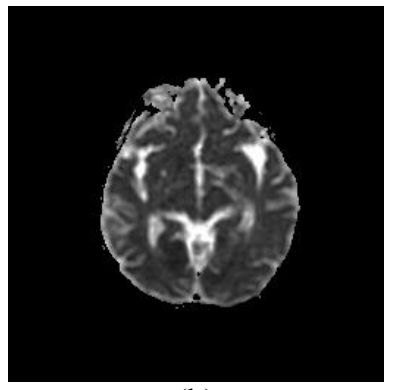

(b)

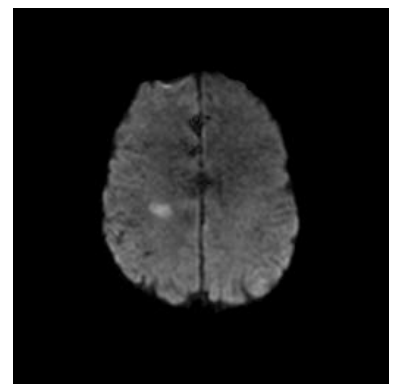

(c)

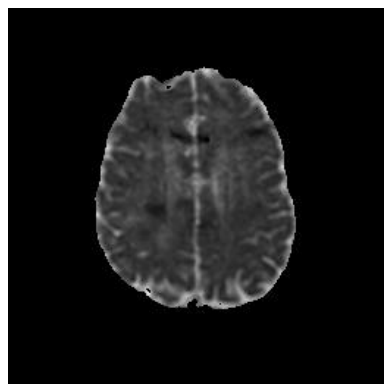

(d) 


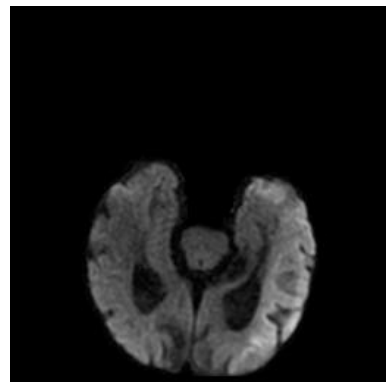

(e)

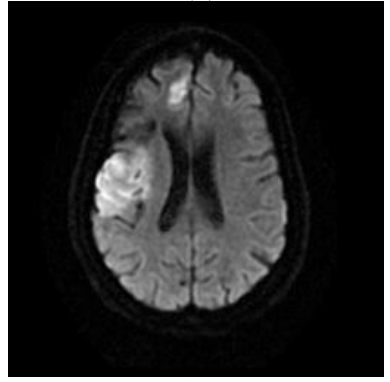

(i)

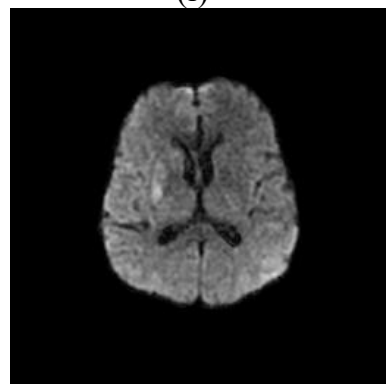

(m)

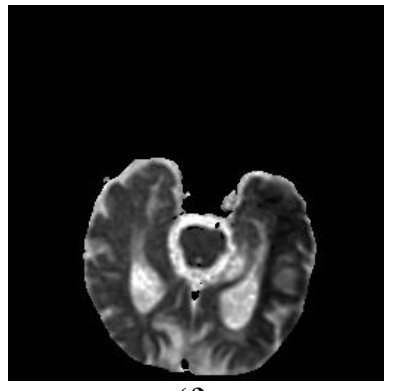

(f)

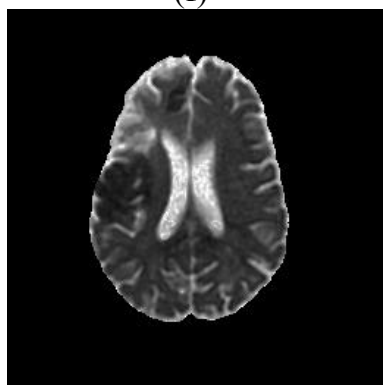

(j)

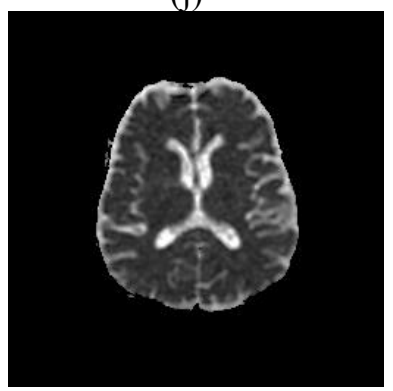

(n)

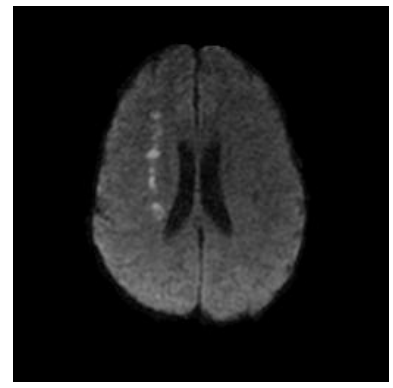

(g)

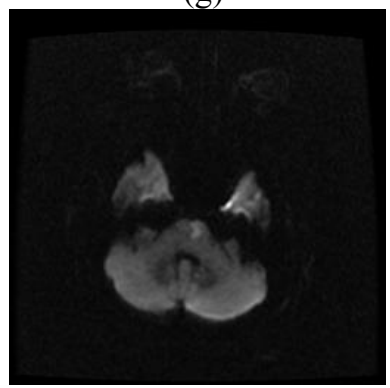

(k)

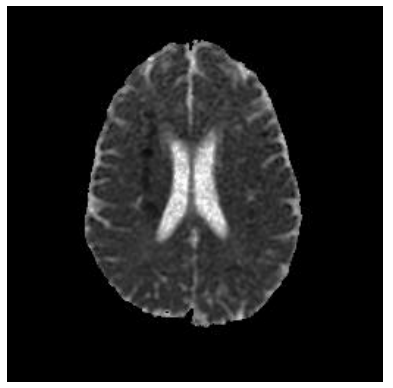

(h)

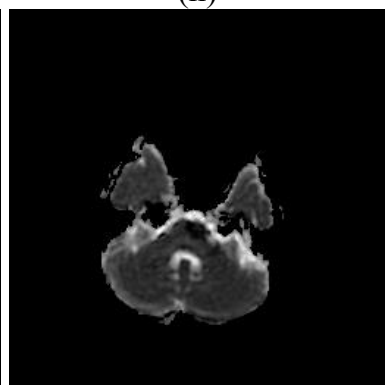

(1)

Figure 1. Input DWI images and ADC maps depicting common infarct patterns: DWI images (a, c, e, g, I, k, m) and corresponding ADC maps $(b, d, f, h, j, l, n)$

\subsection{Relative Difference Map (RDM) - Based Segmentation Algorithm}

A novel algorithm was developed that quantified the degree of relative difference between corresponding regions in the ipsilateral and contralateral hemispheres of the brain. A Relative Difference Map (RDM) $[10,11]$ representing this quantification in 2D was constructed. Subject to a line of symmetry, we use the RDM technique to highlight asymmetric areas in the stroke region in both DWI images and corresponding ADC maps. This is a novel approach that automatically identifies the asymmetry and quantifies the amount of asymmetry. The method automatically identifies the focal points; derives the axis of reflexional symmetry and computes the RDM. For the RDM, we fix a pair of symmetric windows, that are ordered sets of points, of size M by M, about the axis of symmetry and compute the set of relative differences with the associated statistics (mean and variance) while scanning simultaneously both hemispheres. We highlight regions by retaining the original pixel intensities that show relative differences (in the stroke region) and suppressing regions, setting the pixel values to zero, which express symmetric similarity. Such pre-segmented data is ready to serve as input to additional selected segmentation methods. 
To improve the results further and segment stroke regions captured in both DWI images and ADC maps, we utilize a recently developed RDM-based segmentation method designed to quantify and segment of brain pathologies in any images with reflexional symmetry [12]. The RDM method automatically identifies and delineates brain pathology by analyzing the opposing sides of the brain. Because brain exhibits high level of bilateral symmetry and this symmetry is distorted in the presence of pathology, we utilize the inherent left-right symmetry in the brain and use the healthy side of the brain as our prior template to statistically enhance the differences in high dimensional feature space. We replace the prior of a generic statistical normal human brain atlas with a subjectspecific left-to-right symmetry information derived from the subject's brain images. In other words, this method can essentially replace the need for a digital atlas with the cross-registration between opposing hemispheres of the brain.

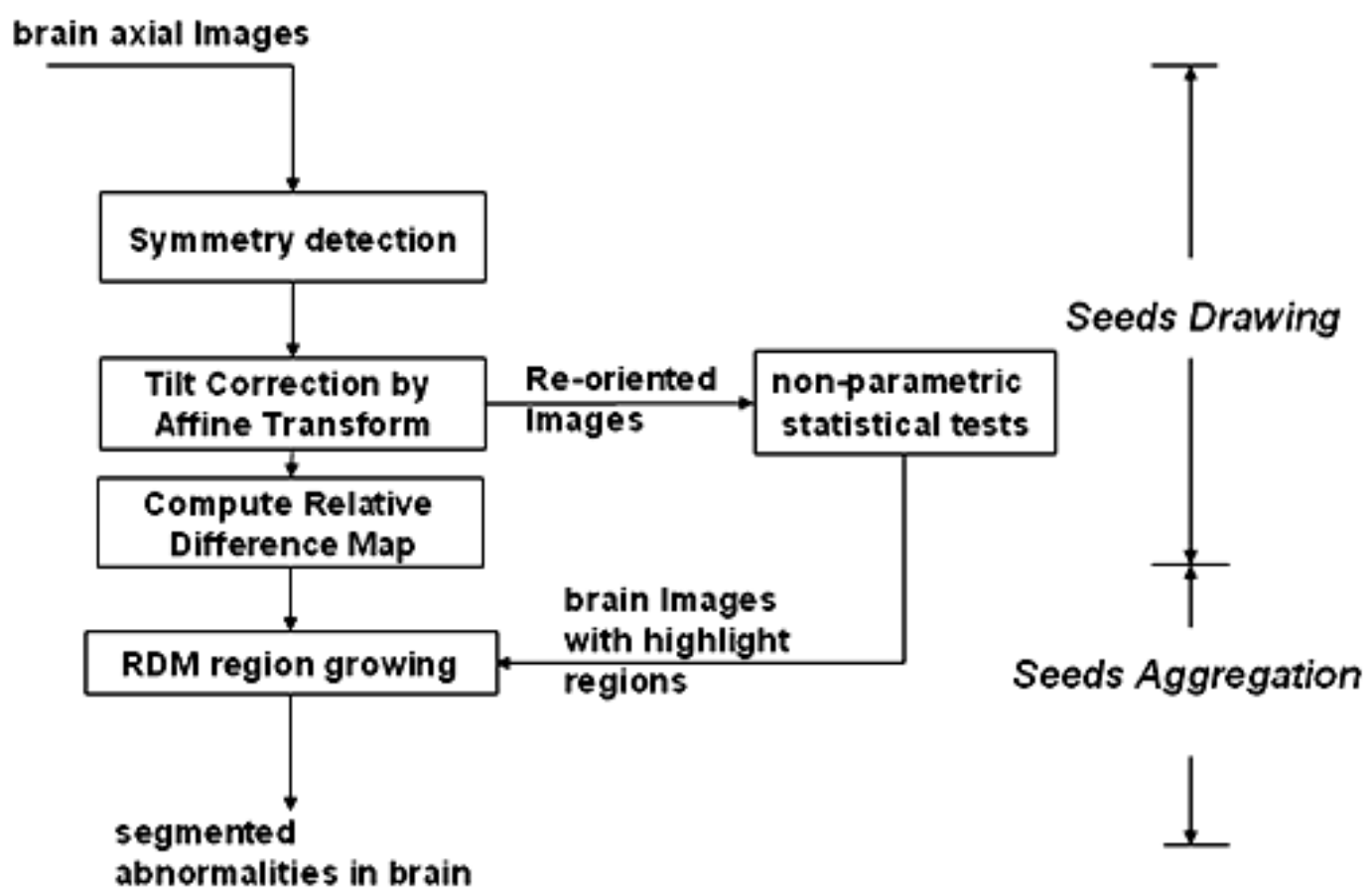

Figure 2. Flow chart of RDM-based segmentation method.

The flowchart of the RDM method is depicted in Figure 2. In the first step, the symmetry axis is automatically computed and the tilt of the head is corrected by applying standard affine transform. The detection of slight variations in the left to right brain imagery is complicated by normal differences in the anatomical structures occurring in the data set. Therefore, we utilize the apparent bi-lateral symmetry in brain imagery through the application of non-parametric statistical tests operating on the pairs of samples and non-parametric statistical tests on local averages. This technique can highlight regions that can be further examined in a "statistical" sense. The highlighted region can be used as "seeds" for later propagation in the difference map that quantified differences between the brain hemispheres $[10,11]$. The statistical tests provide the "likelihood" of the difference to appear randomly given the samples that have a 1:N chance of occurrence without 
an underlying difference being present. Finally, a region is grown within the difference map yielding a segmentation of an abnormal target in the brain.

As shown in Figure 2, the major components of our proposed segmentation pipeline, consists of two consecutive steps, seeds drawing and seeds aggregation. Seeds drawing is composed of the following sub-components: symmetry detection, tilt correction and asymmetry measurement by non-parametric statistical tests. Seeds drawing in our method is fully automated unlike most current region based segmentation approaches. Seeds are understood as the occurrence of regions with statistically minimal chances of an underlying symmetry being present. In radiological brain images, to measure differences in bi-fold mirror symmetry, the axis of symmetry must be computed and the image "self-registered" to this axis. After detection and registration of the image to the axis of symmetry, the data can be checked for significant differences in the image in regions collocated relative to the given axis of symmetry. This approach defines the essence of the relative difference map (RDM) method [10,11], which quantifies and highlights asymmetric areas in the two opposing brain hemispheres. In this paper, we refine the method of selecting an axis of symmetry and computation of relative asymmetry with respect to the axis of symmetry. Our method allows for the computation of the RDM for DWI and ADC maps. For further details describing the technique, see reference [12]. In Figure 3, we show an example MR DWI image and segmented stroke region.

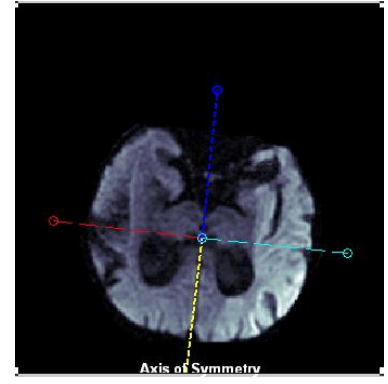

(a)

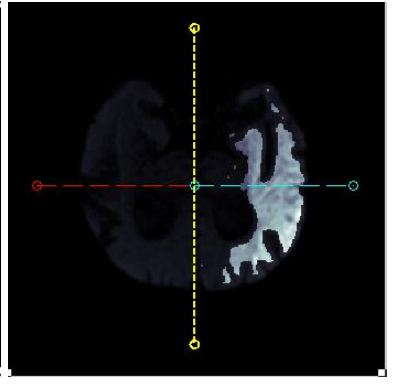

(b)

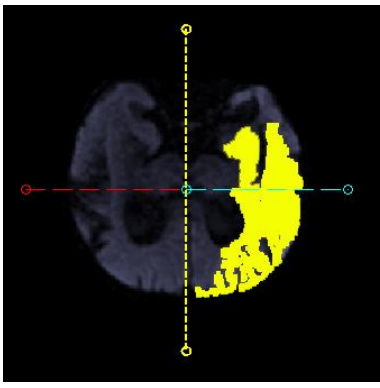

(c)

Figure 3. Segmentation of stroke patient on MR DWI. (a) Original input image; (b) Re-oriented image with identified significant difference; (c) Final segmented region after the RDM-based segmentation.

\subsection{RDM Algorithm Evaluation}

Two experts generated ground truth (GT) for the seven selected cases. Accuracy measurements, FPVF, FNVF, TPVF, [14], were calculated for 1) expert 1 GT compared with RDM delineated DWI infarcts, 2) expert 2 GT compared with RDM delineated DWI infarcts, and 3) RDM delineated DWI infarcts compared with RDM delineated ADC infarcts.

\section{RESULTS}

We performed quantification and delineation of stroke regions, using the RDM-based segmentation method, to process the DWI images and its corresponding ADC maps (see Figure 4). On DWI, some regions with normal diffusion coefficient can also appear as hyperintense. The ADC maps can confirm the presence or absence of an acute infarct by depicting those areas of true diffusion while eliminating T2 and other factors that may affect the signal. A true acute infarct with high signal intensity on DWI will remain hypointense on ADC map [4]. However, ADC maps are 
usually not used alone because of poor signal contrast. Hypointense infarcts tend to be less apparent to the radiologist using ADC maps alone. Thus, DWI and ADC maps are used as complementary techniques in clinical practice. The final indication of the presence of an acute infarct comes from a pre-segmentation of the hypertintense region in the DWI image and the corresponding hypointense region on the ADC map. The accurate delineation of the shape of the stroke region is not our primary focus; rather, we would like to be able to make a binary decision about the presence or absence of an infarct by the presence of delineated overlapping DWI and ADC stroke regions. Such binary results may provide a hint to the physician about the presence of acute stroke. This hint can assist the physician in determining the final diagnostic decision about the patient.

We performed an evaluation of the accuracy of the RDM-based segmentation method. Accuracy denotes the degree to which the segmentation agrees with the ground truth [13]. As part of the accuracy assessment, three different values were obtained: 1) true positive volume fraction (TPVF) is the fraction of the total amount of tissue in the ground truth which overlaps with the experimental method delineation, 2) false positive volume fraction (FPVF) is the fraction of tissue falsely identified by the experimental method and 3) false negative volume fraction (FNVF) is the fraction of tissue in the ground truth that was missed by the experimental delineation method. The accuracy measurement values are shown in Tables 2, 3. Table 2 lists the accuracy values for Expert 1 ground truth as compared to the RDM DWI segmentation algorithm. Table 3 lists the accuracy values for Expert 2 ground truth as compared with the RDM DWI segmentation algorithm. The results from Table 2 and Table 3 indicate that the RDM does accurately delineate the acute infarct region from the corresponding DWI image in all cases except for image set \#7. Image set \#7 differs from the other image sets presented in this study in that it represents a possible TIA (Transient Ischemic Attack) that is a reversible condition and not a complete stroke.

We compared for each patient case the level of overlap of the RDM delineated DWI and corresponding RDM delineated ADC regions. Table 4 lists accuracy measurements for RDM-based segmentation algorithm using the DWI image as compared with the RDM-based segmentation algorithm using the ADC image. The TPVF value is of particular interest to the identification of acute infarcts within DWI images and ADC maps, because a nonzero value of TPVF (the maximum value is 1) indicates presence of an acute infarct. For each of the image sets containing an acute infarct, TPVF values were all greater than zero. There is an open issue how to link the values of non-zero TPVF to the level of acuteness of an infarct. For that, we will have to process hundreds of cases and correlated clinical history to the degree of overlap between the DWI and ADC regions. For example, a patient with acute multiple sclerosis (MS) might have multiple lesions that could show hypointense distributed regions in the ADC maps. Those distributed regions will be segmented as such by our RDM method. There is a chance that an MS lesion from the set will overlap with a nonzero DWI region for the patient, but the value of the resulting TPVF parameter should be very low. Thus, a small TPVF may indicate that there is no acute infarct but rather a MS lesion. For now, we state that any non-zero value of the TPVF should indicate to a neuroradiologist that a high risk of acute infarct exists and that further evaluation of the images will be necessary to arrive at a final diagnosis.

Also, not all small ADC regions are yet detectable by our RDM-based segmentation method. For example, image set \#6 was a small pontine infarct in which the RDM method sensitivity needs improvement. Image set \#7 was the only case in which the TPVF value was zero. Image set \#7 represents a possible TIA. In DWI-positive TIA patients, ADC abnormalities are less pronounced 
than in stroke patients [14]. This method of automated quantification of the level of overlap between DWI and ADC RDM-segmented stroke regions can be a potentially clinically significant result since there is a narrow therapeutic window to treat ischemic strokes (thrombolytics are most effective within 3-6 hours after onset of symptoms). A rapid test that gives a binary hint about the presence/absence of an acute infarct in a patient with suspected stroke should help busy diagnostic radiologists and clinicians to rapidly administer appropriate treatment to a patient.

\begin{tabular}{|c|c|c|c|c|c|c|}
\hline $\begin{array}{l}\text { IMAGE } \\
\text { SET \# }\end{array}$ & $\begin{array}{l}\text { Expert1 } \\
\text { GT AREA }\end{array}$ & $\begin{array}{l}\text { RDM (DWI) } \\
\text { SEGMENTATAION } \\
\text { AREA }\end{array}$ & AREA DIFFERENCE (\%) & FNVF & FPVF & TPVF \\
\hline 1 & 460 & 430 & 4.86 & .1836 & .1349 & .8163 \\
\hline 2 & 114 & 97 & 14.91 & .2017 & .0526 & .7982 \\
\hline 3 & 3636 & 3244 & 50.588 & .1796 & .0718 & .8204 \\
\hline 4 & 255 & 384 & 2.82 & .4039 & .9098 & .5960 \\
\hline 5 & 1487 & 1445 & N/A & .0915 & .0632 & .9085 \\
\hline 6 & 63 & N/A & 94.11 & N/A & N/A & N/A \\
\hline 7 & 102 & 198 & & .5 & 1.441 & .5 \\
\hline
\end{tabular}

Table 2. Accuracy measurements for Expert 1 ground truth (GT) as compared with the RDM DWI segmentation algorithm.

\begin{tabular}{|c|c|l|c|c|c|c|}
\hline $\begin{array}{l}\text { IMAGE } \\
\text { SET \# }\end{array}$ & $\begin{array}{l}\text { Expert 2 } \\
\text { GT AREA }\end{array}$ & $\begin{array}{l}\text { RDM (DWI) } \\
\text { SEGMENTATATION } \\
\text { AREA }\end{array}$ & $\begin{array}{l}\text { AREA DIFFERENCE (\%) } \\
\text { FNVF }\end{array}$ & FPVF & TPVF \\
\hline 1 & 452 & 430 & 4.86 & .1836 & .1349 & .8163 \\
\hline 2 & 114 & 97 & 14.91 & .2105 & .0614 & .7894 \\
\hline 3 & 3162 & 3244 & 2.59 & .1382 & .1641 & .8618 \\
\hline 4 & 153 & 384 & 150.9 & .1830 & 1.692 & .8169 \\
\hline 5 & 1399 & 1445 & 3.29 & .0993 & .1322 & .9006 \\
\hline 6 & 76 & N/A & N/A & N/A & N/A & N/A \\
\hline 7 & 103 & 198 & 92.23 & .5048 & 1.427 & .4951 \\
\hline
\end{tabular}

Table 3. Accuracy measurements for Expert 2 ground truth (GT) as compared with the RDM DWI segmentation algorithm.

\begin{tabular}{|c|c|c|c|c|c|c|}
\hline $\begin{array}{l}\text { IMAG } \\
\text { E SET } \\
\#\end{array}$ & $\begin{array}{l}\text { RDM } \\
\text { (DWI) } \\
\text { GT AREA }\end{array}$ & $\begin{array}{l}\text { RDM (ADC) } \\
\text { SEGMENTATION AREA }\end{array}$ & $\begin{array}{l}\text { AREA DIFFERENCE (\%) } \\
\text { FNVF }\end{array}$ & FPVF & TPVF \\
\hline 1 & 430 & 215 & 50 & .6581 & .1581 & \\
\hline 2 & 97 & 376 & 287.63 & .0206 & 2.896 & .9793 \\
\hline 3 & 3244 & 2875 & 11.37 & .2987 & .1849 & .7012 \\
\hline 4 & 384 & 215 & 44.01 & .5781 & .1380 & .4218 \\
\hline 5 & 1445 & 1499 & 3.74 & .2062 & .2435 & .7937 \\
\hline 6 & N/A & N/A & N/A & N/A & N/A & N/A \\
\hline 7 & 198 & 0 & 100 & 1 & 0 & 0 \\
\hline
\end{tabular}

Table 4. Accuracy measurements for RDM DWI segmentation algorithm as compared with the RDM ADC segmentation algorithm.

Below, we demonstrate our preliminary results on our seven sample images. We are currently processing a large dataset with hundreds of patient cases that are part of an IRB approved retrospective stroke study using DWI images and corresponding ADC maps.

In Figure 4, we present the original images along with the RDM-based segmentation processed files and the two expert generated ground truth. 


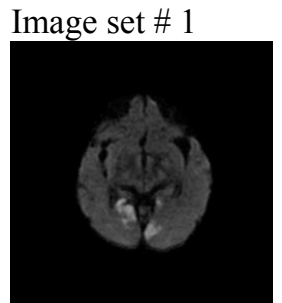

(1)

Image set \#2

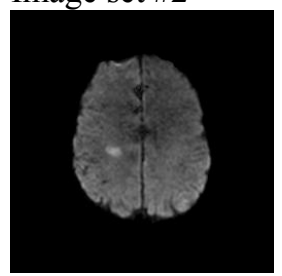

(1)

Image set \#3

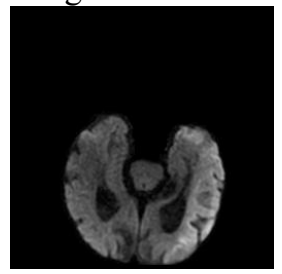

(1)

Image set \#4

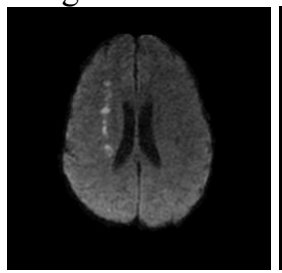

(1)

Image set \#5

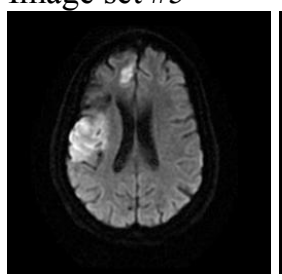

(1)

Image set \#6

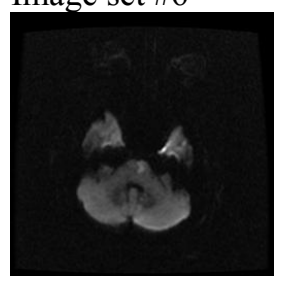

(1)

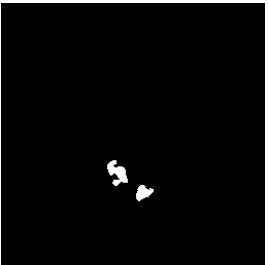

(2)

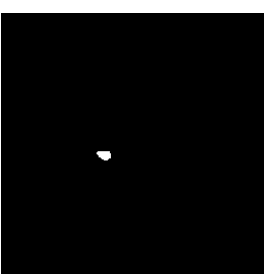

(2)

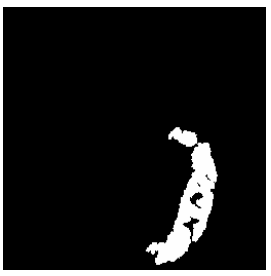

(2)

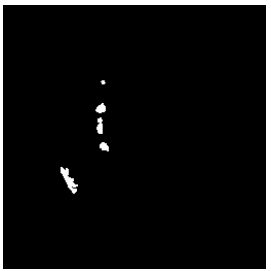

(2)

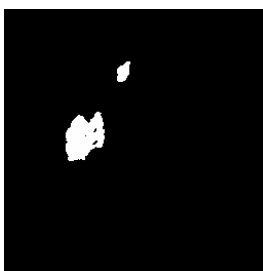

(2)

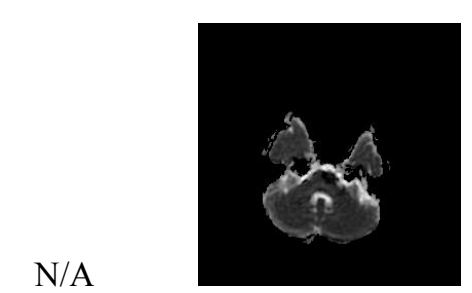

(2)

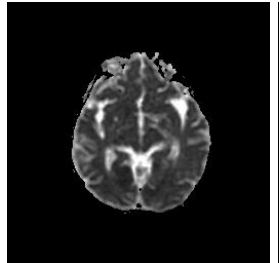

(3)

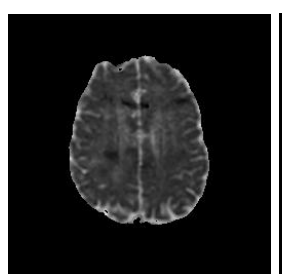

(3)

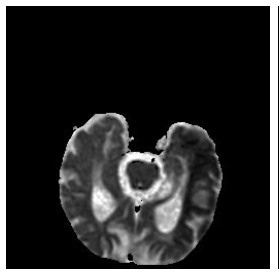

(3)

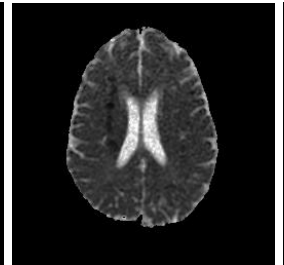

(3)

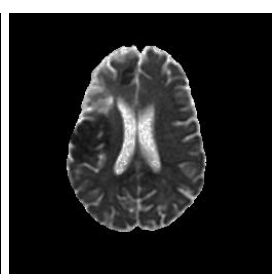

(3)

(3)

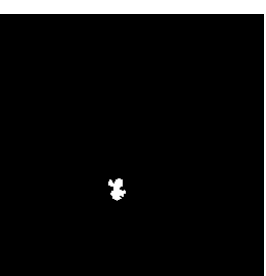

(4)

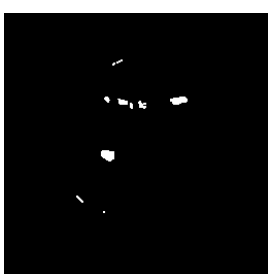

(4)

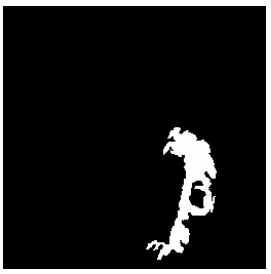

(4)

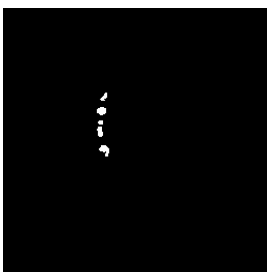

(4)

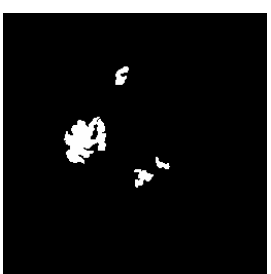

(4)

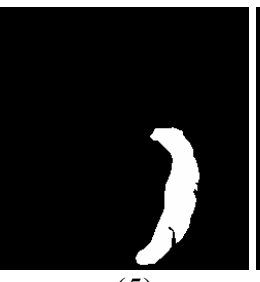

(5)

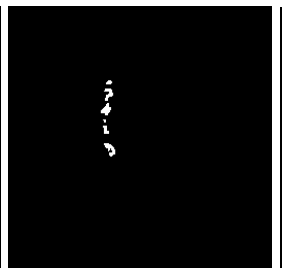

(5)

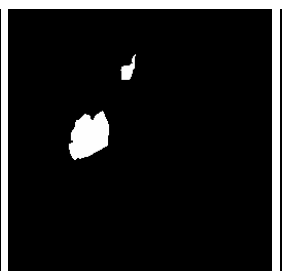

(5)

(5)

(6)

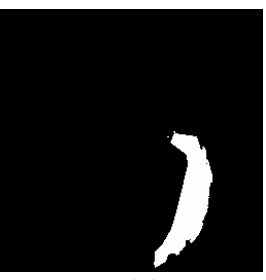

(6)

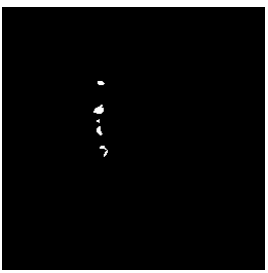

(6)

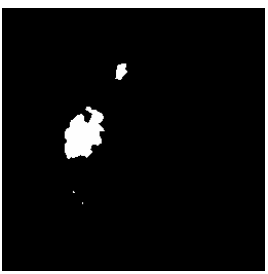

(6)

Proc. of SPIE Vol. 6143 61430T-9 


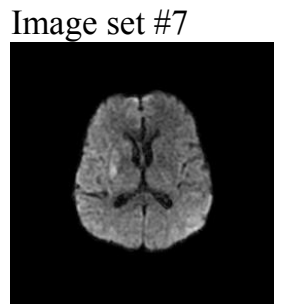

(1)

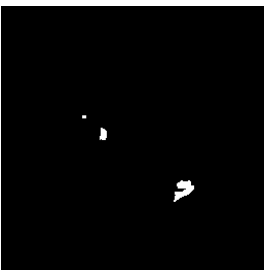

(2)

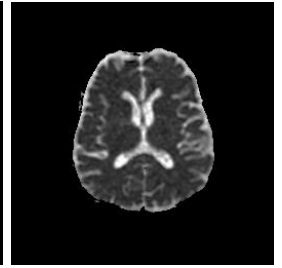

(3)

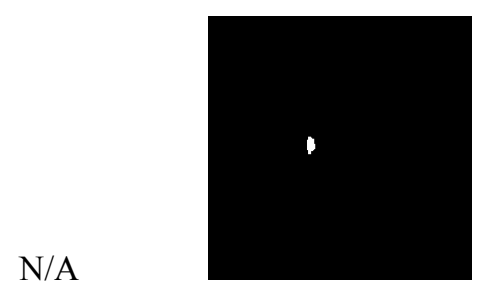

(5)

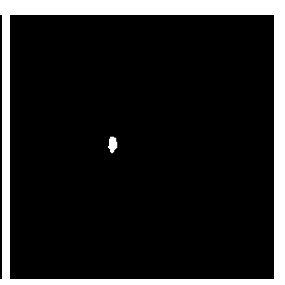

(6)

Figure 4. Original, RDM processed and Ground Truth Images: DWI (1), RDM DWI segmentation area (2), ADC map (3), RDM ADC segmentation area (4), Expert 1 delineated DWI Ground Truth (5) and Expert 2 delineated Ground Truth (6) for all seven sets. Set \#6 with a very small pontine infarct was difficult to process with the RDM method.

We are currently increasing the sensitivity of our method to handle images with small pontine infarcts, like the one in case \#6. If we can resolve delineation of this small infarct region in the DWI image and its corresponding ADC map, this clinically challenging case could be detected and quantified by our method to assist radiologists.

\section{CONCLUSIONS}

We present a novel RDM method to quantify and delineate infarct regions in DWI images and corresponding ADC maps. The accuracy of automated RDM-based segmentation was evaluated using expert delineated ground truth. The amount of overlap was computed between RDMsegmented infarct regions in DWI images and corresponding ADC maps.

Creating a computer aided diagnostic tool that would provide a binary clue about the absence or presence of an acute infarct would greatly contribute to improved patient outcomes by triaging a large number of patient image datasets for the radiologist. Such a tool should be evaluated using a large clinical data set in order to validate the binary classifier. The tool should also be tested in a clinical setting to evaluate its performance and the radiologist observer performance using ROC (receiver operating characteristic) methodology [15]. Moreover, ROC curves would measure the performance levels of the radiologists both with and without computer aid.

Diffusion weighted images, although very rapid, can still be susceptible to motion. Movement of a patient's head during the imaging process causes apparent motion of all the pixels in the image. Motion introduces phase errors during acquisition that leads to motion artifacts affecting the quality of the images [16]. A number of studies have attempted to address the motion issue through the use of various image sequence enhancements, such as propeller DWI, and other processing techniques.

The focus of this preliminary study is on the methodology required to process DWI and ADC maps for delineating infarct regions. Future research will involve an analysis of motion. In addition to motion, patients' have a tendency to tilt their heads during a long scanning session causing an asymmetry in the left and right brain structures. Future work will describe the effect of such asymmetry on the methodology described above, and will evaluate the performance of the methodology after applying an affine transform as a solution to compensate for patient tilt. 


\section{REFERENCES}

[1]. Smajlovic D, Sinanovic O. Sensitivity of the neuroimaging techniques in ischemic stroke. Med Arh. 2004;58(5):282-4.

[2]. van Everdingen KJ, van der Grond J, Kappelle LJ, Ramos LM, Mali WP. Diffusion-weighted magnetic resonance imaging in acute stroke. Stroke. 1998 Sep;29(9):1783-90.

[3]. Mori S B, P.B. Diffusion Magnetic resonance Imaging: Its Principles and Applications. Anat Rec. 1999 Jun 15;257(3):102-9.

[4]. Roberts TP, Rowley HA. Diffusion weighted magnetic resonance imaging in stroke. Eur J Radiol. 2003 Mar;45(3):185-94.

[5]. Mullins ME, Schaefer PW, Sorensen AG, Halpern EF, Ay H, He J, et al. CT and conventional and diffusionweighted MR imaging in acute stroke: study in 691 patients at presentation to the emergency department. Radiology. 2002 Aug;224(2):353-60.

[6]. Calamante F, Gadian DG, Connelly A. Quantification of perfusion using bolus tracking magnetic resonance imaging in stroke: assumptions, limitations, and potential implications for clinical use. Stroke. 2002 Apr;33(4):1146-51.

[7]. Rordorf G, Koroshetz WJ, Copen WA, Cramer SC, Schaefer PW, Budzik RF, Jr., et al. Regional ischemia and ischemic injury in patients with acute middle cerebral artery stroke as defined by early diffusion-weighted and perfusion-weighted MRI. Stroke. 1998 May;29(5):939-43.

[8]. Braun J BJ, Koennecke H, Wolf K Automated Tissue Characterization in MR Imaging. Proc SPIE. 2000;3978:32-45.

[9]. Martel A, Alder SJ, Delay GS, Morgan PS, Moody AR. Measurement of Infarct Volume in Stroke Patients using Adaptive Segmentation of Diffusion Weighted Images. MICCAI '99 Lecture Notes in Computer Science. 1999;1679:22-31.

[10]. Imielinska C, Liu X, Rosiene J, Sughrue ME, Komotar RJ, Mocco J, et al. Toward objective quantification of perfusion-weighted computed tomography in subarachnoid hemorrhage: quantification of symmetry and automated delineation of vascular territories. Acad Radiol. 2005 Jul;12(7):874-87.

[11]. Imielinska C LX, Sughrue M, Hagiwara E, Connolly ES, D'Ambrosio A. Objective Quantification of Perfusion-Weighted Computed Tomography in the Setting of Subarachnoid Hemorrhage. Computer Assisted Radiology and Surgery. 2004 June:34-43.

[12]. Liu X, Imielinska C, Rosiene J, Connolly ES, D'Ambrosio A. Enchanced Techniques for Asymmetry Quantification in Brain Imagery. SPIE Conference on Medical Imaging 2006. 2006.

[13]. Udupa JK LV, Schmidt H, Imielinska C, Saha PK, Grevera GJ, Zhuge Y, Currie LM, Molholt P, Jin Y. .

Methodology for evaluating image-segmentation algorithms. SPIE Conference on Medical Imaging. 2002;4684:266-77. [14]. Kidwell CS, Alger JR, Di Salle F, Starkman S, Villablanca P, Bentson J, et al. Diffusion MRI in patients with transient ischemic attacks. Stroke. 1999 Jun;30(6):1174-80.

[15]. Metz CE. ROC methodology in radiologic imaging. Invest Radiol. 1986 Sep;21(9):720-33.

[16]. Miller KL, Pauly JM. Nonlinear phase correction for navigated diffusion imaging. Magn Reson Med. 2003 Aug;50(2):343-53. 